\title{
Mapping dynamic, non-Euclidean spaces
}

\author{
Nick Lally ${ }^{\mathrm{a}}$, Luke Bergmann ${ }^{\mathrm{b}}$ \\ ${ }^{a}$ Department of Geography, University of Kentucky, nicklally@uky.edu \\ ${ }^{b}$ Department of Geography, University of British Columbia, luke.bergmann@ubc.ca \\ * Corresponding author
}

Keywords: space, creative cartography, relational, non-euclidean

\begin{abstract}
:
Space is often described as a dynamic entity in human geographic theory, one that resists being pinned down to static representations. Co-produced in and through relations between various things and phenomena, space in these accounts is variously described as being contingent, processual, plastic, relational, situated, topological, and uneven. In contrast, most cartographic methods and tools are based on static, Euclidean understandings of space that can be reduced to a simple, mathematical description. In this work, I explore how cartography can deal with space as a dynamic and fluid concept that is entangled with the phenomena and objects being mapped. To those ends, I describe a method for creating animated maps based on relational understandings of space that are always in flux.
\end{abstract}

This work builds on research in collaboration with Luke Bergmann, where we suggest a move from Geographic Information Systems (GIS) as we commonly know them to the broader realm of geographical imagination systems (gis) that are informed by spatial theory in human geography. The animated maps here are produced using our prototype gis software Enfolding, which use multidimensional scaling (MDS) to visualize relational spaces, in combination with Blender, an open-source 3D rendering program. Written in JavaScript and available as open source software ${ }^{1}$, Enfolding is our first attempt to make gis an accessible set of tools that expand the possibilities for mapping by providing new grammars for creative cartographic practices.

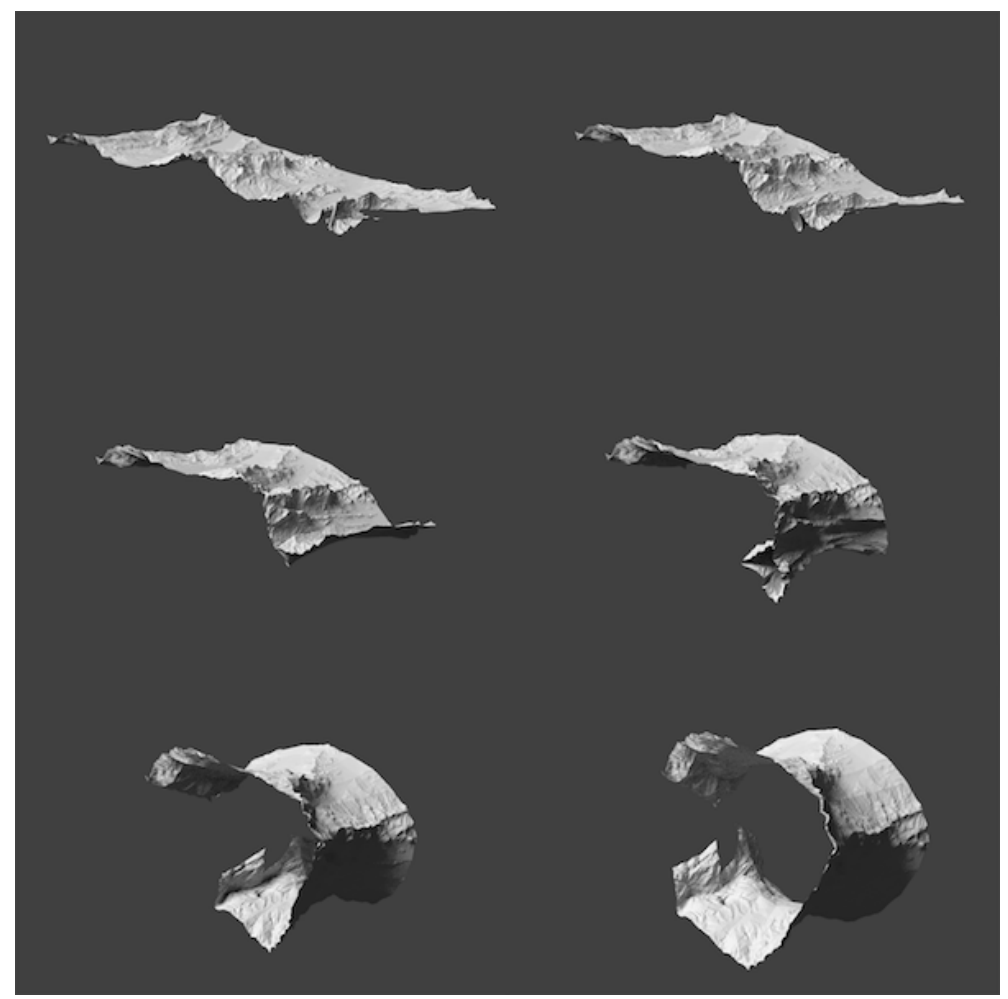

Figure 1. Animation stills.

In the cartographic workflow presented here, I use Enfolding to produce manifolds from a set of points and userdefined distances between points. Changing those measures of distance-which might represent travel times, affective connections, communicative links, or any other relationship as defined by a user-produces shifting manifolds. Using the .obj export option in Enfolding, I then import the manifolds into Blender, using them as animation keyframes. In

\footnotetext{
${ }^{1}$ Users can view and modify the codebase on GitHub: https://github.com/FoldingSpace/enfolding
} 
Figure 1, I have added a digital elevation model (DEM) to the 3D figure, producing an animated visualization of a dynamic and relational space that includes a hillshade.

This workflow represents only one of many creative possibilities for innovative cartographic practices that engage with space as a matter of concern. With growing interest in 3D cartographic methods comes expanded possibilities for visualizing dynamic and relational spaces. Combining conceptual antecedents in both human and quantitative geography with current cartographic methods allows for new approaches to both mapping and space. The workflow and tools that have emerged from this research are presented here with the hope of spurring creative and exploratory cartographic work that draws from but also contributes to vibrant discussions in spatial theory and creative cartography. 